\title{
LA RELACIÓN ENTRE LOS ACTORES SOCIALES LA PARTICIPACIÓN Y EL CONTEXTO
}

\section{Cecilia Inés Zapata López'}

"La actuación entendida como una actividad artística tendría que comprender que el actor no es un ilustrador, no debería ser un ilustrador de ideas de otros sino que tiene que poder escribir con su propio trazo una escritura en paralelo a la del texto y el director." ${ }^{2}$

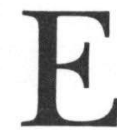

n la última década tomó fuerza la utilización del término actor(es) social(es) en el ámbito de las prácticas sociales; pero es difícil encontrar una definición clara al respecto, se utiliza cada vez que se requiere nombrarlos y hacer alusión a ellos, sin detenerse en apreciaciones que posibiliten su caracterización y clasificación sin ningún tipo de exclusión.

El propósito del artículo es iniciar una búsqueda hacia la claridad del término actor social y relacionarlo con otros como: agentes sociales, institución, profesional de trabajo social y usuario, que tradicionalmente se han utilizado en la profesión de trabajo social, para referirse a quienes participan en procesos de interacción en un mismo escenario y contexto, con determinados fines hacia la búsqueda de mejores opciones de desarrollo.

Puede concluirse de los textos que utilizan dicho término, que siempre se refieren a personas que son o deben ser consideradas sujetos del desarrollo económico y social en un país y por lo tanto, con poder de participación en la sociedad a la cual pertenecen. Se

\footnotetext{
${ }^{1}$ Docente e investigadora en la Universidad de Antioquia, Medellín, Colombia.

${ }^{2}$ SPREGELBURD, RAFAEL (2000, 4 de marzo). El actor es el verdadero productor de sentido. El Mundo, Medellín, págs. 4 - 5, c. 2.
} 
encuentran dos elementos de importancia para que el trabajador social profundice en la claridad de dicho concepto, el primero como posibilidad de dinamizar su espacio profesional en interlocución con otras profesiones afines, el segundo para profundizar en su objeto de intervención profesional.

Haciendo una revisión acerca del significado del término actor social, se puede deducir que su connotación, es heredada del mundo teatral y mezclada con la realidad social; para adquirir una dimensión nueva se requiere precisar la definición y así llegar a un proceso de naturalización en las profesiones que vienen utilizando el término.

Otras veces se ha criticado la utilización del término actor social, por considerar equivocadamente, que este lleva implícito el sello de ser un objeto utilizado y en este sentido perdería la capacidad que, para el trabajador social, tienen las personas individualmente, en grupos, familias o comunidades, de ser consideradas autónomas y sujetos sociales. Al respecto, se encuentran, dentro del teatro, opiniones que derrumban esa creencia:

se habla del actor como objeto, del actor sin yo, del actor como modeloplastilina-... Los buenos actores son los que han escapado a esa supuesta profesión de actor. El actor tiene un trazo de la misma manera que el pintor tiene un trazo o que el músico tiene un trazo; pero el actor se ha puesto durante mucho tiempo al servicio del realismo que le exige la narración y ha olvidado esa capacidad que tiene de ser él el verdadero productor de sentido (Spreguelburd, 2000, pág. 4).

Se considera pertinente luego de esta introducción y antes de continuar el desarrollo del tema acerca de los actores sociales, contexto y participación, tratar de definir el término actores sociales, como los individuos, grupos, familias, comunidades y organizaciones formales o informales con capacidad real o potencial de incidir en su propio desarrollo, en inter-relación con otros grupos y con el Estado.

El tema de la relación entre los actores sociales, la participación y el contexto, será abordado desde tres ángulos, a saber:

- Relación entre agentes sociales y actores sociales

- Prácticas sociales y contexto

- Los actores sociales y la participación como opción de modificación del contexto. 
La variable primera, es importante porque los sujetos sociales que están en interacción, pueden ser llamados de acuerdo a la época y el contexto de diferentes formas. Esto se debe a la dinámica que tienen todos los lenguajes y es significativo que el profesional de trabajo social se acerque al estudio de dichas modificaciones en la búsqueda de precisión de su lenguaje especializado. El sub-tema de prácticas sociales y contexto, cobra vigencia porque es el escenario en el que se mueven los actores sociales, a quiénes se hará referencia. Finalmente, la interacción de esos actores con el medio social en el que están inmersos, exige una propuesta metodológica para la intervención profesional, que para este caso se eligió entre otras posibilidades, la participación.

Se considera que precisar y profundizar cada vez más en las variables propuestas para el desarrollo de este artículo, pueden constituir una forma de mirar la práctica de trabajo social, con todos los elementos que están en juego en cualquier intervención profesional, posibilitando una visión general para ese quehacer profesional que debe ser integrador, sin descuidar la especialidad. (Ver Gráfica en anejo 1)

\section{Relación entre agentes sociales y actores sociales}

Tradicionalmente, en la profesión de Trabajo Social, para hacer referencia al trabajo con todo tipo de población, se hablaba de: individuo, grupo y comunidad; pero luego se vio la necesidad de hablar de: individuo, grupo, comunidad y familia. También, para referirse a todos los espacios profesionales de interacción, se alude a tres agentes sociales: la institución, el trabajador social y el usuario (Tobón, s.f.).

En el agente profesional, es decir en el Trabajador Social, ha de tenerse en cuenta su formación profesional, lo cual va respaldado por la academia y el saber popular, es decir todas las ciencias, teorías y saberes. Se habla de saberes, como un reconocimiento de que no solo el académico es quien produce teoría, también se da como producto de la interrelación entre la academia y las experiencias de las personas que protagonizan cotidianamente esa realidad social en la cual se trabaja.

En lo que la profesión tradicionalmente ha llamado el agente institucional, va incluido tanto lo gubernamental como no gubernamental, encontrando en los últimos actores sociales, que, dada la coyuntura sociopolítica que atraviesa el país, han recobrado el 
reconocimiento e importancia, para que se conviertan en dinamizadores de los procesos de cambio requeridos en la sociedad y en cooperación con el Estado. Estos agentes institucionales pueden agruparse así:

- Movimientos Sociales

- Organismos no gubernamentales

- Empresa Privada

- Partidos Políticos

\section{Prácticas sociales y contexto}

Los actores sociales considerados como sujetos de su propio desarrollo, son necesarios en la construcción de su sociedad, por esto es interesante detenerse en el tema para lograr una comprensión holística y transdisciplinaria de su papel en los procesos comunitarios participativos, que permitan tomar conciencia de lo global y lo local a nivel planetario, desde las relaciones intra e interdependientes de diferentes esferas contextuales, definidas no solamente por territorios sino por dinámicas específicas de acuerdo a su propia historia.

Los actores sociales, están inmersos en un contexto de la sociedad a la que pertenecen y cuando se habla de ella, hace referencia a un resultado de múltiples interrelaciones entre individuos, grupos formales e informales, la familia como grupo primario por excelencia y comunidades. Ello también implica que estén en un territorio definido por ellos mismos, que puede ir desde, el ámbito familiar, vecinal, sectorial, barrial, comunal, zonal, de ciudad, regional, departamental, nacional, continental, hasta el planeta en que vivimos.

Pero la sociedad como un todo es compleja de abordar para un análisis de contexto, lo que sugiere una mirada a sus organizaciones y comunidades, permitiendo así unidades de análisis más accesibles en la búsqueda de significados. Se entenderá por comunidad aquella organización social resultante de un proceso, donde individuos y grupos comparten actividades y objetivos comunes que posibilitan un sentido de pertenencia a ella y que pueden o no compartir un territorio común, caracterizada por fuertes lazos de solidaridad, cooperación y ciertas garantías de permanencia en ella, por tiempos que pueden ir hasta de generación en generación. 
Es precisamente en la sociedad y en las comunidades donde se puede analizar el contexto y el encuentro de diferentes esferas de relación, cuyo punto de encuentro ha de ser una sociedad civil organizada, por medio de los diferentes actores sociales identificados previamente y que son quienes van a posibilitar unas prácticas sociales acordes con sus intereses y aspiraciones, generando proyectos de vida individuales y colectivos, que entrelazados de una comunidad a otra, pueden acercarse o no a un proyecto común de sociedad.

Los espacios de interacción como, los movimientos sociales, organismos no gubernamentales, partidos políticos, empresa privada, individuos, grupos, familias y comunidades, constituyen un campo amplio de trabajo, donde intencionalmente se puede llegar a definir qué sociedad se quiere y si existe o no, disposición a trabajar por ella y hasta donde se quiere hacer compromiso o ser respuesta a lo que el contexto exige, lo que implica participar. Cuando se habla de un proyecto común de sociedad, no se quiere decir con ello que es homogénea, se trata de permitir la diversidad y pluralidad, en un clima democrático donde los actores sociales sean capaces de buscar puntos de trabajo conjunto, sin abandonar intereses específicos.

Es importante resaltar la existencia de trabajos o prácticas sociales realizadas por algunos actores sociales en la búsqueda de generar procesos y modificaciones al contexto, aunque hay que reconocer que dentro de ellos también se continúa con la perpetuación de muchas prácticas que no inciden en cambio alguno. De forma breve se hace referencia a algunos de ellos, lo cual amerita en otro momento profundización. Por ejemplo, en los movimientos sociales encontramos, a grupos de base, movimientos ecológicos, grupos de mujeres, de jóvenes, de ancianos, etc, y son un ejemplo de movilización hacia el cambio, con fuertes lazos de pertenencia, sin que ello implique estructuras jerárquicas rígidas, pues al contrario, se caracterizan por ser descentralizadores. También se destaca en ellos el pluralismo, lo que permite que se unan grupos con diferentes intereses alrededor de causas comunes que requieren la unión de sus fuerzas.

Los partidos políticos también, constituyen otros actores sociales que en Colombia, por la necesidad de iniciar con el rompimiento de los esquemas tradicionales partidistas, se ha dado proliferación de matices de los mismos y se ha ido buscando la representatividad de grupos poblacionales específicos que antes eran marginados, 
lo cual evidencia la necesidad de los gobiernos a respetar y tener en cuenta la diferencia y pluralidad de la población. La existencia de grupos políticos múltiples genera la necesidad de interactuar entre ellos para llegar a acuerdos, hacer coaliciones o replantear viejas costumbres que no responden a las necesidades de una nueva sociedad.

Respecto a las organizaciones no gubernamentales, consideradas como entidades sin ánimo de lucro que desean participar en la construcción de una nueva sociedad y en algunos casos llenan vacíos del Estado, han proliferado en los últimos años, llegando algunas a abanderar importantes proyectos comunitarios de diversa índole y en otros casos reflejando el continuismo del asistencialismo, que fue una característica en sus inicios. Actualmente se ha visto la necesidad de intervenciones coordinadas de estas organizaciones con el Estado y en muchos casos se trabaja por contrataciones del último a las primeras.

La empresa privada requiere convertirse en actora social protagónica, con una función social, ya que no puede aislar su función económica, del bienestar requerido por sus empleados y la población en la cual está inmersa, considerada como una comunidad con la cual interactúa de forma directa o indirecta y que por lo tanto se afectan mutuamente.

Existe la teoría acerca de la empresa y sus relaciones con los "stakeholders", es decir, los grupos comprometidos que le apuestan al éxito de la organización (Vázquez, 2000). El modelo conceptual consiste en considerarla como un campo de fuerzas generado por la interacción de tres elementos básicos que son: empleados, accionistas y clientes. Dentro de esta concepción cada uno aporta recursos y expectativas y reclama beneficios y derechos. Además, están otros tres constitutivos grupos básicos que son: sociedad, gobierno y proveedores, todos contenidos dentro de una relación de intercambio transaccional donde la generación de riqueza por la empresa, es el factor sine qua non que genera el cumplimiento de las expectativas de los grupos constitutivos y, por ende, el crecimiento y desarrollo.

En la teoría planteada, la responsabilidad de la empresa es retribuir adecuadamente las expectativas de los grupos y aprovechar los recursos y contribuciones que cada uno provee. Involucra también el concepto ético de la empresa, que tiene tres niveles: el primero es de cumplimiento con la reglamentación legal exigida; el segundo, ser respuesta social y contribuir más allá de lo que la 
ley exige y el tercero implica que la empresa sea un actor social comprometido con el desarrollo de la comunidad, localidad, región o nación.

Respecto a la academia, como actor social involucrado en las prácticas sociales y contexto, también se encuentra en un replanteamiento de su responsabilidad en las comunidades, debido a que se estaba alejando del servicio al hombre y su realidad, que ha de ser precisamente el sentido de la teoría y sus saberes. No solamente han de estar representados, en quienes ejercen el poder dentro de la academia, sino que además, debe estar representado en los pobladores, en los líderes formales e informales, en profesionales habitantes de las mismas comunidades, en profesionales externos a ellas y en instituciones locales, regionales, nacionales e internacionales.

Retomando todo lo dicho hasta ahora, puede decirse que se presenta una mezcla de actores sociales, intercambiando sus experiencias, donde los últimos buscan directa o indirectamente el apoyo de todas las ciencias, sus teorías y saberes, para integrarlo a sus prácticas sociales. Al hablar de todas las ciencias, no solo hace referencia a aquellas que se relacionan con las sociales y humanidades, debido a que aún aquellas áreas del saber que aparentemente no tienen que ver con la conducta y comportamiento de la sociedad, están ejerciendo influencia y es allí donde el Pensamiento Complejo nos aporta explicando la necesidad de hacer una lectura de la realidad de forma más globalizante, lo que requiere no parcializar las interpretaciones y respuestas.

También está la sabiduría dada por la experiencia de líderes informales, habitantes, estudiosos y personajes que desean que la vida cotidiana no quede relegada a una simple rutina y pase a convertirse en algo dinámico, que cobre vida y sentido en un marco de referencia que haga relación a una historia y a un aprendizaje de ésta. Esta sabiduría generalmente no se encuentra escrita, está en las historias orales, en el pensamiento de los gestores y ejecutores de proyectos concretos realizados en las comunidades con la participación de organizaciones formales e informales a las cuales se hizo referencia. Se considera la sabiduría como una resultante de experiencias y comprensiones que se convierten en una forma de ser y de actuar expresadas en conductas individuales o comunitarias, que a su vez abren nuevos campos de posibilidades para plasmar los proyectos de vida. 


\section{La participación como opción para modificar el contexto.}

Para comprender la ubicación de los actores sociales en un contexto planetario, debe comprenderse el movimiento dinámico que está al interior de los actores sociales organizados o no, los cuales empiezan a interactuar con el Estado y con todas las teorías y saberes, produciendo un campo común donde están entremezclados todos los tipos de actores sociales posibles, como resultado de las interacciones que van de lo micro a lo macro y en forma de red, que posibilita un resultado sinérgico, difícil de dimensionar cuando no existe construcción colectiva de todos los actores sociales implicados.

El proyecto de sociedad que han de construir los actores sociales, considerados éstos como principales protagonistas y sujetos conscientes de sus aspiraciones e intereses, está enmarcado dentro de un gran contexto dado por el planeta tierra, que según Morín (1995) debe llegar a ser nuestra Patria y es por esto que es apropiado hablar de un contexto planetario, el cual implica pensar en lo bio-psicosocial, cultural, económico, político y espiritual.

Profundizando en el contexto y su relación con la participación, se hace alusión a la relación del hombre con la naturaleza, consigo mismo, con la civilización, su historia, sus modos de producción, su posición ideológica y con lo espiritual. Esto último vale la pena diferenciarlo del concepto de religión, para darle una connotación que trascienda los sistemas de creencias y dogmas y llegue a la relación que el hombre establece en un sentido holístico, integrador, globalizante, acerca de su Ser y el sentido que quiere dar a su existencia en el grupo, familia, comunidad, patria, planeta o cosmos.

Haciendo referencia a ese contexto planetario en el cual están todos los actores sociales que trabajan en proyectos de nuevas sociedades, se encuentra lo siguiente:

El constante movimiento del cosmos obedece a una intención. Este orden y esta intención escapan a nuestra limitada comprensión, a no ser que cambiemos la concepción mecánica por una más integral y universal que nos relacione con todos los fenómenos existentes (Programa de Naciones Unidas para el Desarrollo, 1993, pág. 10).

Es importante que aunque se hable de hechos concretos como los presentados entre los diferentes actores sociales y el Estado, sean ubicados en su contexto planetario, para no olvidar que todo está en interrelación y nada se presenta por azar, lo cual implica que 
exista armonía de todas las partes. El mayor ejemplo de lo último podemos hallarlo en la naturaleza cuando no se presenta alterada por el hombre, como en el ciclo de reproducción animal y en el universo cuando los planetas giran alrededor del sol sin chocar unos con otros; queda por trabajar en procura de esa armonía, con todos esos aspectos que tienen que ver con el tipo de relaciones establecidas por el hombre en la búsqueda de su bienestar integral, lo cual solo se logrará cuando cada actor social se ubique y actúe de acuerdo a su papel en este tejido social.

Todos los actores sociales y su relación con el contexto, generan una realidad concreta con aciertos y desaciertos, los cuales pueden ser o no modificados de acuerdo al papel que quieran jugar los primeros. Es por ello que cuando se decide cambiar, puede hablarse de que existen unas acciones o prácticas sociales las cuales pueden ser realizadas por medio de procesos de: investigación, diagnóstico, planeación, ejecución, control y evaluación. Cuando se habla de investigar, diagnosticar o conocer la realidad, existen riesgos relacionados con el cuestionamiento acerca de qué es realmente lo real, porque hay que ser conscientes de que el acercamiento a ésta, exige una visión transdisciplinaria, una conciencia multidimensional, de lo contrario los análisis son simplistas, recortados y unidimensionales; por lo tanto nadie debe tener la posibilidad de creer que tiene el saber total porque "la totalidad es la no verdad" (Marín, 1995, pág. 101).

Planteada la necesidad de actuar y comprometerse, quedan por elgir alternativas posibles de acuerdo a los objetivos buscados y más concretamente a los proyectos que quieran realizar, es ahí donde están los actores sociales y la aplicabilidad que hacen a sus teorías y saberes. También se plantean estrategias y metodologías de trabajo, las cuales pueden tener características de directivas o participativas, pero en el caso planteado, donde los actores sociales son sujetos de su propio desarrollo, existe la posibilidad de la participación en cualquiera de sus modalidades: social y comunitaria (desde lo privado), ciudadana y política (desde lo público), ya que de lo contrario algunos estarían decidiendo por todos.

Otro aspecto bien interesante de estudiar a nivel individual y colectivo, tiene que ver con el significado de participar y lo que implica. Este debe ser motivo de reflexión en cada quien, sin embargo, puede hablarse algo respecto a la participación sin entrar en definiciones que pueden ser consultadas en otros textos. Ante todo, 
antes de optar por la participación es importante tener un nivel de comprensión hacia la necesidad de vivir en relación con otros, en el contexto de la sociedad y más concretamente de la comunidad, donde cada cual evoluciona, física, emocional, cultural, económica, política y espiritualmente.

Clarificar porqué es importante trabajar con otros, permite la capacidad de compartir metas y objetivos comunes, lo cual unido a un concepto holístico permitirá que se desee la construcción de una sociedad más humanizada, democrática, pluralista. Ello implica que si se quiere trabajar en -un proyecto para una nueva sociedad- deba asumir retos y compromisos no solo a nivel personal sino con otros, y esto es participar.

Así que participar, es opuesto a individualismo como tendencia del hombre a considerarse el propio centro, constituyéndose en un concepto comunitario, implica compartir y aportar a otros mediante respuestas concretas que trasciendan el simple discurso. También posibilita el reconocimiento de la necesidad de trabajar en equipo y transdisciplinariamente, fomentando la solidaridad y cooperación. Participar es abrir las puertas a todo un campo de posibilidades para la creatividad y el rompimiento de paradigmas, es construir en compañía la propia historia y la de la sociedad, es permitir convertirse en actor social protagonista, sujeto del desarrollo.

Para poder participar se requiere que conozca a nivel individual y comunitario cuáles son las dinámicas endógenas y exógenas, ya que ello facilitará saber con que potencialidades se cuenta para la participación y cuáles hacen falta para optimizarla. Además, es importante conocer los tipos de interacción dados entre lo micro y lo macro de todos los contextos; para abrir las posibilidades hacia una nueva sociedad, trabajando por proyectos de vida comunitarios.

Este concepto de actores sociales como sujetos de su propio desarrollo puede ser ampliado con los aportes de la teoría del Desarrollo a Escala Humana, la cual abre nuevas posibilidades con sus conceptos de: desarrollo, necesidades humanas, autodependencia, microorganizaciones y relaciones entre la Sociedad Civil y el Estado.

Respecto al planteamiento anterior, rompe con el concepto de desarrollo desde el punto de vista de crecimiento económico y plantea un desarrollo más humano que busca mejorar la calidad de vida de las personas. Esto requiere la satisfacción de las necesidades humanas fundamentales, de ser, tener, hacer y estar; dentro de un 
marco de referencia que involucra a las personas desde una visión holística. También establece la relación y armonía que debe darse entre las personas y la sociedad, más concretamente entre Sociedad Civil y Estado (Max Neef y otros, 1986).

La interacción de los actores sociales, de los cuales ya se ha hecho referencia en los párrafos anteriores:

3.1. Individuos, grupos, familias y comunidades.

3.2. Los movimientos sociales, empresa privada, organismos no gubernamentales, partidos políticos.

3.3. Las teorías y saberes y

3.4. El Estado.

Son cuatro instancias, con un dinamismo expresado en sus prácticas sociales, que desborda su análisis en un contexto planetario (bio-psicosocial, cultural, político, económico y espiritual), que ha influido para que surja la necesidad de un Desarrollo a Escala $\mathrm{Hu}$ mana y a su vez éste retroalimenta el contexto dado (Ver Gráfica en Anejo 1). También puede visualizarse por el análisis realizado, que existe un actor social constante y es la persona que actúa a nivel individual o colectivo, pero para que pueda lograr un desarrollo armónico no sólo con él mismo, sino con el contexto en el cual vive, requiere organización por medio de la participación en la construcción de proyectos comunitarios con intereses específicos. Lo anterior en un marco de pluralismo, respeto a la diversidad y visión de futuro, posibilitará que se pueda contar con una nueva sociedad.

En síntesis, los actores sociales son quienes generan una realidad social y su contexto, siendo a su vez, el último modificador de la conducta del primero; sin embargo, es requisito que los actores sociales como sujetos de su propio desarrollo asuman alternativas que posibiliten la modificación del contexto. Una opción planteada es la que tiene que ver con la participación, como forma de intervenir en el cambio del mismo, de acuerdo a los proyectos individuales o colectivos elegidos por los actores sociales. 


\section{Bibliografía}

Bejarano, Ana María (1992). Democracia y sociedad civil. Análisis político (15), enero-abril.

Fals Borda, Orlando (1990). El papel político de los movimientos sociales. Revista Foro (11).

González, Esperanza (1995). Manual sobre participación y organización para la gestión local. Colombia: Ediciones Foro.

Max Neef, Manfred y otros (1986). Desarrollo a Escala Humana una opción para el futuro Development Dialogue, [Número Especial], 92.

Morín, Edgar (1995). Introducción al pensamiento complejo.(Trad. Marcelo Packman). Barcelona: Editorial Gedisa.

Morín, Edgar y Kern, Anne Brigitte (1993). Tierra Patria. (Trad. Ricardo Figueira). Buenos Aires: Ediciones Nueva Visión.

Múnera, María Cecilia (1994). Hacia un desarrollo no convencional. Medellín: Universidad Nacional, CEHAP, Centros de Estudios del Hábitat Popular.

Programa de las Naciones Unidas para el desarrollo y otros (1993). La Armonía Cósmica, Nuestro Compromiso con la Tierra. Medellín: Editorial Cadena.

Riechmann, Jorge y Fernández Buey, Francisco (1994). Redes que dan libertad. Barcelona: Editorial Paidos.

Spregelburd, Rafael (2000, marzo 4). El actor es el verdadero productor de sentido. El Mundo, Medellín, pp. 4-5.

Tobón, María Cecilia, Rottier, Norma y Manrique, Antonieta (s.f.). La práctica profesional del Trabajador Social. Perú: CELATS. 
LA RELACIÓN ENTRE LOS ACTORES SOCIALES...

Vargas C., Hernán y otros. (1992). Acerca de la naturaleza y evolución de las Ongs en Colombia. Bogotá, Fundación Social.

Vásquez Castro, Orlando. (2000). La empresa y sus grupos comprometidos, Cali, Colombia: Universidad Icesi y Autónoma. 


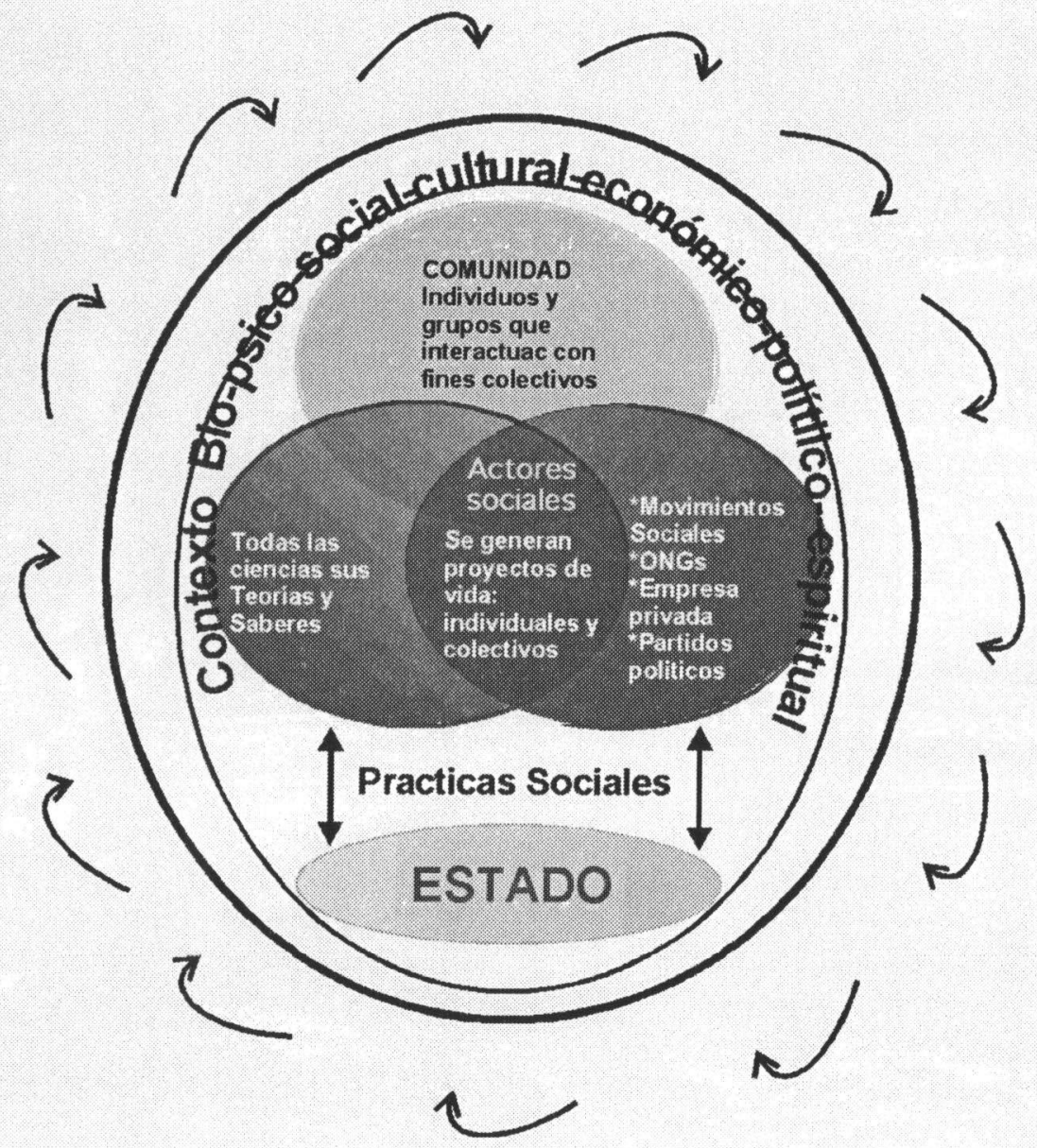

